\title{
ХАРАКТЕРИСТИКИ УПРАВЛЕНИЯ ОДНОФАЗНОГО ДИФФЕРЕНЦИАЛЬНО-ТРАНСФОРМАТОРНОГО МАГНИТНОГО УСИЛИТЕЛЯ
}

Характеристики управления однофазного дифференциально-трансформаторного магнитного усилителя (1ТМУ) или, по определению некоторых исследователей, магнитного удвоителя частоты с самоподмагничиванием, изучались только в некоторых предельных условиях $\left[{ }^{1,2}\right]$. В настоящей статье эти характеристики рассматриваются в более общем режиме, который содержит ранее .известные режимы как частные случаи.

Анализ 1ТМУ проводится при следующих основных допущениях: . кривая намагничивания соответствует приведенной на рис. 1, б, активные сопротивления равны нулю, число витков всех обмоток одинаково, вентиль идеальный.

a

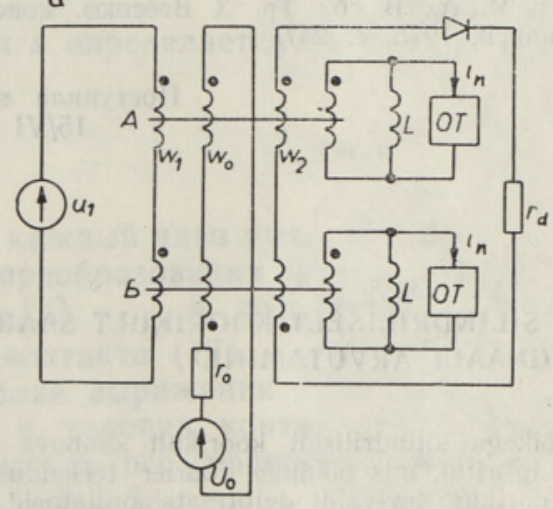

$\delta$

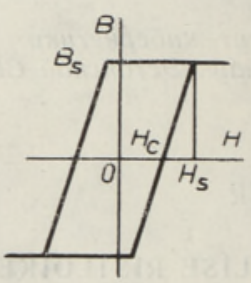

Рис. 1.

При этих упрощениях с учетом преобразования $\left[{ }^{3,4}\right]$ получим схему 1 ТМУ, показанную на рис. $1, a$. Основные параметры схемы следующие:

$$
\begin{gathered}
L=\frac{w^{2} S}{l} \cdot \frac{B_{s}}{H_{s}-H_{c}}, \\
I_{\mathrm{I}}=\frac{H_{\mathrm{c}} l}{w}, \\
U_{1 m} \leqslant 2 \omega S w B_{s},
\end{gathered}
$$

где $w$ - число витков обмоток, $S$ - поперечное сечение сердечника, $l$ - средняя длина линии магнитной индукции, $B_{s}$ - индукция насы- 
щения, $H_{s}-$ напряженность магнитного поля насыщения, $H_{c}-$ коэрцитивная сила, $\omega-$ угловая частота напряжения питания. Остальные обозначения ясны из рис. $1, a$. Ограничитель тока ОТ характеризует гистерезис $\left(\operatorname{sign} i_{\Pi}=\operatorname{sign} u_{1}\right)$.

Условие (3) обозначает, что из рассмотрения исключаются режимы с одновременным насыщением сердечников. В данной статье принимается

$$
U_{1 m}=2 \omega S w B_{s} .
$$

Приведенная на рис. 1, а схема весьма неудобна для анализа. На рис. 2 ей соответствует преобразованная в $\left[{ }^{5}\right]$ схема замещения, в которой параметры сердечников и мощности элементов оставлены без изменений, а ветвь намагничивания, не влияющая на работу схемы, опущена.

Қак показано в [ $\left.{ }^{1}\right]$, источники переменного напряжения в схеме на рис. 2 можно заменить источниками напряжения удвоенной частоты

$$
e_{s}^{\prime}=-e_{q}^{\prime}= \begin{cases}-\frac{U_{1 m}}{2} \cos \omega t, & 2 n \pi \leqslant \omega t \leqslant(2 n+1) \pi, \\ -\frac{U_{1 m}}{2} \cos \omega t, & (2 n+1) \pi \leqslant \omega t \leqslant(2 n+2) \pi .\end{cases}
$$

С целью упрощения расчетов в схеме замещения устранены цифровые коэффициенты, то есть напряжения увеличены в два $\left(e_{s}=2 e_{s}^{\prime}\right)$ и сопротивления в четыре раза. При этом соответствие электромагнитных процессов сохраняется, а мощность всех элементов увеличивается в четыре раза (в данном случае это несущественно).

Расчетная схема приведена на рис. 3. Известно $\left[{ }^{1}\right]$, что при указанной на рис. 3 полярности напряжения управления $U_{0}$ работает только магнитный ключ $k_{s}$ и ток нагрузки $i_{d}$ не влияет на характеристики управления 1ТМУ. Поэтому ветви с $e_{q}\left(k_{q}\right)$ и $i_{d}$ здесь не рассматриваются.
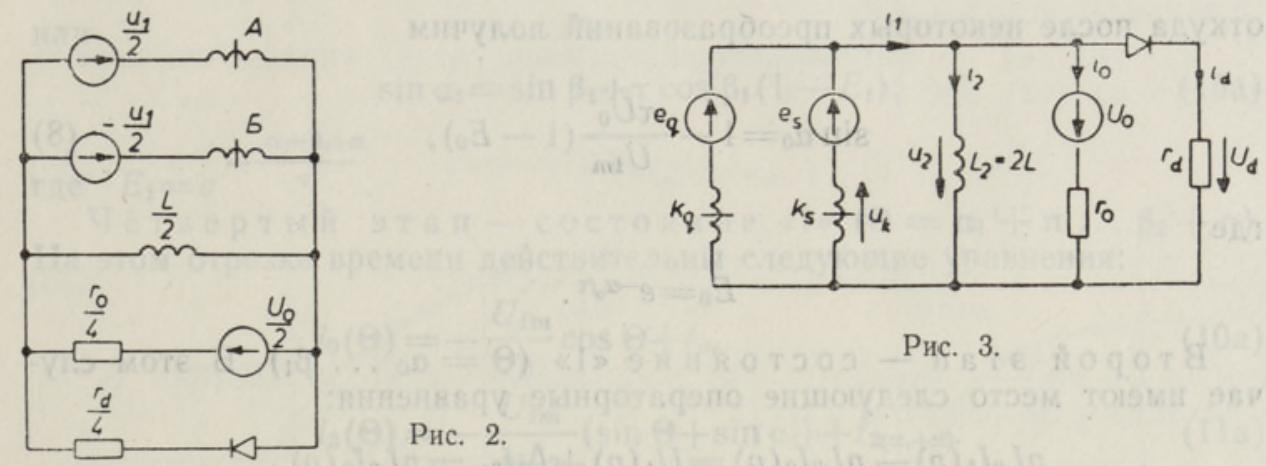

Рис. 3.

Рис. 2.

При смене полярности $U_{0}$ (в данной статье также не исследуется) работает только ключ $k_{q}$ и $i_{d}$ способен существенно изменять эти характеристики.

Следовательно, замыкание (насыщение сердечника) и размыкание ключа $k_{s}$ (выход сердечника из насыщения) возможны при следующих условиях:

$$
\int_{\beta}^{\pi+\alpha} u_{k} d \Theta=0
$$




$$
i_{1 \beta}=0,
$$

где $\boldsymbol{\alpha}$ - угол замыкания ключа, $\beta$ - угол размыкания ключа, $u_{k}-$ напряжение на ключе, $\Theta=\omega t$.

Проанализируем работу схемы по этапам. Каждому этапу соответствует определенное состояние схемы. Замкнутое состояние будем называть состоянием «1» и разомкнутое - состоянием «0».

Для схемы на рис. 3 в состоянии «0» действительно следующее операторное уравнение

$$
\left(p L_{2}+r_{0}\right) I_{0}(p)=U_{0}(p)-L_{2} I_{20}
$$

Принимая начальный ток $I_{20}=0$, получим

$$
\begin{aligned}
& i_{0}(\Theta)=\frac{U_{0}}{r_{0}}\left(1-e^{-\Theta / \tau}\right)=-i_{2}(\Theta), \\
& u_{2}=-L_{2} \frac{d i_{0}}{d t}=-U_{0} e^{-\Theta / \tau}, \\
& u_{k}=e_{s}-u_{2}=u_{1}-u_{2},
\end{aligned}
$$

где

$$
\tau=\frac{\omega L_{2}}{r_{0}} .
$$

Первый этап- состояние «0» $\left(\Theta=0 \ldots \alpha_{0}\right): u_{1}=$ $=U_{1 m} \cos \Theta, u_{k}=U_{1 m} \cos \Theta+U_{0} e^{-\Theta / \tau}$. Из условия замыкания ключа (5) следует

$$
\int_{0}^{\alpha_{0}} u_{k} d \Theta=2 \omega \Delta \Psi_{1}=2 \omega\left(\Psi_{S}-\Psi_{0}\right)=2 \omega \Psi_{S},
$$

откуда после некоторых преобразований получим

$$
\sin \alpha_{0}=1-\frac{\tau U_{0}}{U_{1 m}}\left(1-E_{0}\right)
$$

где

$$
E_{0}=e^{-\alpha_{0} / \tau}
$$

В торой этап - состояни е $1 »\left(\Theta=\alpha_{0} \ldots \beta_{1}\right)$. В этом случае имеют место следующие операторные уравнения:

$$
\begin{aligned}
& p L_{2} I_{1}(p)-p L_{2} I_{0}(p)=U_{1}(p)+L_{2} I_{2 \alpha_{0}}=p L_{2} I_{2}(p), \\
& \left(p L_{2}+r_{0}\right) I_{0}(p)-p L_{2} I_{1}(p)=U_{0}(p)-L_{2} I_{2 \alpha_{0}}, \\
& I_{1}(p)-I_{0}(p)=I_{2}(p) .
\end{aligned}
$$

Решая систему уравнений (9) и учитывая, что

$$
I_{2 \alpha_{0}}=-\frac{U_{0}}{r_{0}}\left(1-E_{0}\right)=-I_{0}\left(1-E_{0}\right),
$$

получим 


$$
\begin{aligned}
& i_{0}(\Theta)=\frac{U_{1 m}}{r_{0}} \cos \Theta+I_{0}, \\
& i_{2}(\Theta)=\frac{U_{1 m}}{r_{0}}\left(\sin \Theta-\sin \alpha_{0}\right)-I_{0}\left(1-E_{0}\right) .
\end{aligned}
$$

Применяя условие размыкания ключа (6) к уравнениям (10) и (11), можно найти $\beta_{1}=f_{0}\left(\alpha_{0}\right)$, которое преобразуется к виду

$$
\sin \left(\beta_{1}+\varphi\right)=\cos \varphi\left(1-\frac{\tau U_{0}}{U_{1 m}},\right.
$$

где $\varphi=\operatorname{arctg} \tau$.

Третий этап - состояние $« 0 »\left(\Theta=\beta_{1} \ldots \alpha_{1}+\pi\right)$. Соответствующее операторное уравнение

$$
\left(p L_{2}+r_{0}\right) I_{0}(p)=U_{0}(p)-L_{2} I_{2 \beta_{1}},
$$

откуда

$$
i_{0}=I_{0}\left(1-e^{-\frac{\theta-\beta_{1}}{\tau}}\right)-I_{2 \beta_{1}} e^{-\frac{\theta-\beta_{1}}{\tau}} .
$$

Учитывая, что из (11) или (10) можно определить

$$
I_{2 \beta_{1}}=\frac{U_{1 m}}{\omega L_{2}}\left(\sin \beta_{1}-\sin \alpha_{0}\right)-I_{0}\left(1-E_{0}\right)=-\frac{U_{1 m}}{r_{0}} \cos \beta_{1}+I_{0},
$$

и принимая во внимание условие замыкания ключа (5), после некоторых преобразований находим $\alpha_{1}=f_{1}\left(\beta_{1}\right)$ в виде

$$
\sin \alpha_{1}=E_{1} \sin \beta_{1}+1-\frac{\tau U_{0}}{U_{1 m}}\left(1-E_{1}\right)
$$

или

$$
\sin \alpha_{1}=\sin \beta_{1}+\tau \cos \beta_{1}\left(1-E_{1}\right),
$$

где $E_{1}=e^{-\frac{\alpha_{1}-\beta_{1}+\pi}{\tau}}$.

Четверты й эт а п - состояние «1» $\left(\Theta=\alpha_{1}+\pi \ldots \beta_{2}+\pi\right)$. На этом отрезке времени действительны следующие уравнения:

$$
\begin{aligned}
& i_{0}(\Theta)=-\frac{U_{1 m}}{r_{0}} \cos \Theta+I_{0}, \\
& i_{2}(\Theta)=-\frac{U_{1 m}}{\omega L_{2}}\left(\sin \Theta+\sin \alpha_{1}\right)+I_{2\left(\alpha_{1}+\pi\right)} .
\end{aligned}
$$

Учитывая

$$
I_{2\left(\alpha_{1}+\pi\right)}=I_{2 \beta_{1}} E_{1}-I_{0}\left(1-E_{1}\right),
$$

а также выражение (14) для $I_{2 \beta_{1}}$ и условие размыкания ключа (6) при $\Theta=\beta_{2}+\pi$, после преобразований получим

$$
\sin \left(\beta_{2}+\varphi\right)=\cos \varphi\left(1-\frac{\tau U_{0}}{U_{1 m}}\right) .
$$

Из равенств (17) и (12) ясно, что $\beta_{1} \equiv \beta_{2}=\beta$, следовательно, и 
$\alpha_{1} \equiv \alpha_{2}=\alpha$, то есть полученный нами режим является установившимся, а схема на рис. 3 - быстродействующей.

При $r_{0}=\infty$ (соответствует схеме с идеальным источником тока $\left.I_{0}\right)$ из (17) с учетом (3a) получим:

$$
\sin \beta=1-\frac{\omega L_{2} I_{0}}{U_{1 m}},
$$

откуда

$$
\frac{U_{1 m}}{\omega L_{2}} \sin \beta=\frac{\Psi_{S}}{L}-I_{0}=I_{S}-I_{0}
$$

а из (15а) следует

$$
\sin \alpha=\sin \beta,
$$

откуда

$$
\alpha=\pi-\beta .
$$

При $\omega L=\infty$ (кривая намагничивания сердечника идеальная) из (17) получим

$$
\cos \beta=-\frac{U_{0}}{U_{1 m}}
$$

и из (15a)

$$
\sin \alpha-\sin \beta=(\alpha-\beta+\pi) \cos \beta .
$$

Приведенные выше формулы (18)-(21) соответствуют результатам работ $\left[{ }^{1,2}\right]$.

Анализ удобно провести в относительных единицах. Для этого введем следующие понятия.

1. $U_{0 N}\left(I_{0 N}\right)$ - номинальное напряжение (ток) управления определяется из (12) при $\beta=\pi$ :

$$
\begin{aligned}
& U_{0 N}=U_{1 m} \frac{1+\tau}{\tau}, \\
& I_{0 N}=\frac{U_{0 N}}{r_{0}}=\frac{U_{1 m}}{\omega L_{2}}(1+\tau) .
\end{aligned}
$$

2. $U_{0^{*}}\left(I_{0^{*}}\right)$ - относительное напряжение (ток) управления (с использованием формулы (17)):

$$
U_{0^{*}}=\frac{U_{0}}{U_{0 N}}=\frac{U_{0}}{U_{1 m}} \frac{\tau}{1+\tau}=\frac{1}{1+\tau}\left[1-\frac{\sin (\beta+\varphi)}{\cos \varphi}\right]=I_{0^{*}}
$$

3. $U_{d N}-$ номинальное выходное напряжение. Учитывая, что

$$
U_{d}=\frac{1}{\pi} \int_{\alpha}^{\pi / 2} \cos \Theta d \Theta=\frac{U_{1 m}}{\pi}(1-\sin \alpha)
$$

примем 


$$
U_{d N}=\frac{U_{1 m}}{\pi} .
$$

4. $U_{d^{*}}$ - относительное выходное напряжение:

$$
U_{d^{*}}=\frac{U_{d}}{U_{d N}}=1-\sin \alpha .
$$

Таким образом, для определения характеристик управления 1ТМУ имеем следующие формулы:

$$
\begin{aligned}
& \sin \alpha-\sin \beta=(1-E) \tau \cos \beta, \\
& U_{0^{*}}=\frac{1}{1+\tau}\left[1-\frac{\sin (\beta+\varphi)}{\cos \varphi}\right], \\
& U_{d^{*}}=1-\sin \alpha,
\end{aligned}
$$

где $E=e^{-\frac{\alpha-\beta+\pi}{\tau}}$. Расчеты для четырех значений $\tau$ показаны на рис. 4 .

Экспериментальные исследования проводили на диодной модели 1ТМУ $\left[{ }^{6}\right]$ и на реальном 1 ТМУ. Результаты испытаний на диодной модели отличаются от теоретически предсказанных не более чем на $2-3 \%$. Реальный 1 ТМУ имел следующие данные: сердечники из 50 НП 0,05 типа ОЛ 164/110-30, активное сечение $6,64 \mathrm{~cm}^{2}$, средняя длина магнитопровода $0,43 \mu$. Магнитная проницаемость сердечника на восходящей ветви петли гистерезиса $\mu \approx 0,11 \Gamma \mathrm{H} / \mathrm{M}$. Обмотки усилителя были изготовлены из провода ПЭВ-2 1,56 , число витков $w_{1}=500, w_{2}=200$, $w_{0}=$ по выбору.

В первую очередь была определена т. н. естественная характеристика управления. Элементом управления усилителя служил стабилизированный источник постоянного тока, последовательно соединенный с индуктивностью $L_{0}=2,5 \Gamma \mathrm{H}$. По остальным параметрам эксперимента $\left(U_{1}=208 B, w_{0}=5\right.$ витков, $\left.r_{d}=1 \kappa O M\right)$ были определены

$$
\begin{aligned}
& U_{d N}=\frac{\sqrt{2} U_{1} w_{2}}{w_{1} \pi}=37,6 B, \\
& F_{0}=w_{0} I_{0}=5 I_{0} .
\end{aligned}
$$

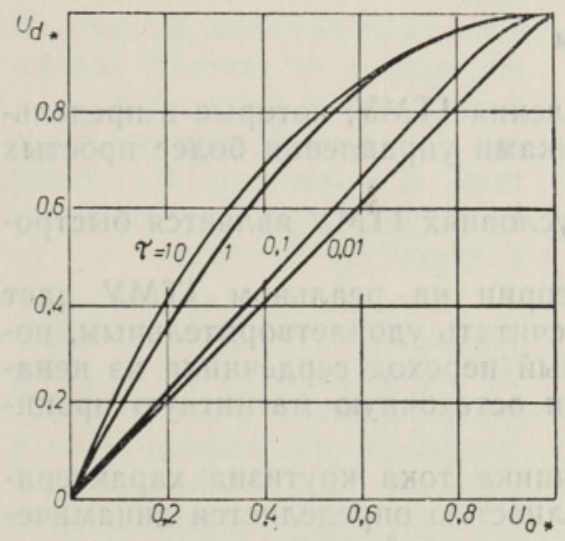

Рис. 4.

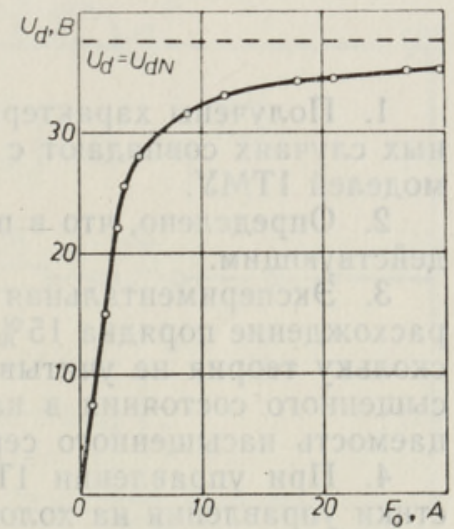

Puc. 5 . 
Полученная характеристика $U_{d}\left(F_{0}\right)$ (рис. 5) соответствует практическому холостому ходу 1 ТМУ $\left(r_{d}=1 \kappa O M\right)$. Как видно из рис. 5 , линейность естественной характеристики сохраняется до $U_{d}=22 B$ $\left(U_{d^{*}}=0,58\right)$. Нелинейность характеристики в остальной части обусловливается плавным переходом сердечника из ненасыщенного состояния в насыщенное. Поскольку теория предполагает неизменную индуктивность обмотки, то совпадения теории с экспериментом следует ожидать на линейном участке естественной характеристики управления, т. е. до $U_{d}=22 B$.

По техническим причинам дальнейшие эксперименты были проведены при $w_{0}=20$ витков. Индуктивное сопротивление обмоток $\omega L_{2}$ определялось с помощью петли гистерезиса и из естественной характеристики управления (рис. 5). С использованием формул (18), (19), (25) и (26) было найдено

$$
\omega L_{2}=\frac{\pi w_{0}}{w_{2}} \cdot \frac{\Delta U_{d}}{\Delta I_{0}},
$$

где $\Delta U_{d}$ - прирост выходного напряжения при изменении тока управления на величину $\Delta I_{0}$. Полученные

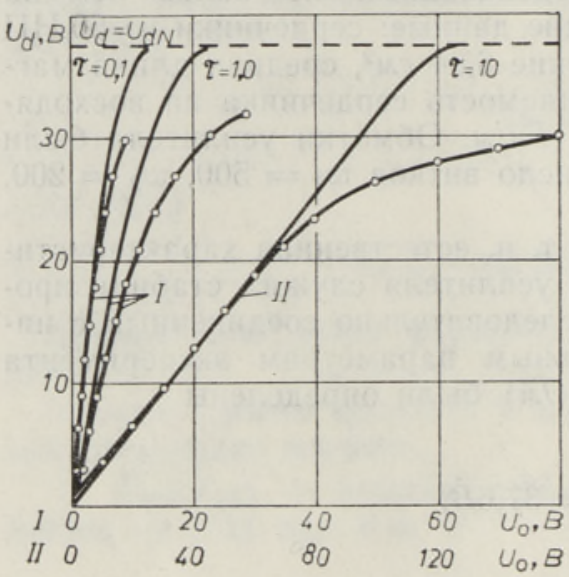

Рис. 6. данные отличались от теоретически предсказанных не более чем на $6 \%$.

Исходя из предварительных измерений сопротивления цепи управления $\tau$ были выбраны равными $10 ; 1,0$ и 0,1 . Результаты экспериментов иллюстрирует рис. 6 , где они сравнены с соответствующими теоретическими характеристиками управления. Как видно, в предполагаемой области совпадения $\left(U_{d}=0-22 B\right)$ хорошее согласие теории с экспериментом наблюдается для $\tau=10$ и 0,1 . Причины удовлетворительного совпадения при $\tau=1,0$ требуют дальнейшего анализа на более сложных моделях 1TMY.

\section{Выводы}

1. Получены характеристики управления 1ТМУ, которые в предельных случаях совпадают с характеристиками управления более простых моделей 1ТМУ.

2. Определено, что в приведенных условиях 1ТМУ является быстродействующим.

3. Экспериментальная проверка теории на реальном 1ТМУ дает расхождение порядка $15 \%$, что можно считать удовлетворительным, поскольку теория не учитывает ни плавный переход сердечника из ненасыщенного состояния в насыщенное, ни остаточную магнитную проницаемость насыщенного сердечника.

4. При управлении 1ТМУ от источника тока крутизна характеристики управления на холостом ходу полностью определяется динамической проницаемостью сердечника нар восходящей ветви петли гистерезиса, 


\section{ЛИТЕРАТУРА}

1. С арв В., О явээр М., Саккос Т., Х ун т Ю., Магнитные преобразователи электроэнергии с удвоением частоты, Таллин, 1972.

2. С а р в В. В., В сб.: Проблемы технической электродинамики, вып. 24, Электромагнитные и полупроводниковые устройства преобразовательной техники, Киев, 1970.

3. Котова Л. Ф., Электричество, № 4, 88 (1967).

4. Розенблат М. А., Магнитные элементы автоматики и вычислительной техники, М., 1974.

5. Л а усм а а Т., Х ун т Ю., Изв. АН ЭССР, Физ. Матем., 25, 192 (1976).

6. С а р в В., Изв. АН ЭССР, Физ. Матем., 21, 264 (1972).

Ннститут термофизики и электрофизики

Академии наук Әстонской ССР ।

Поступила в редакцию

29/IV 1976

\section{KAASIK, J. SOOJARV, J. HUNT, T. LAUSMAA}

\section{UHEFAASILISE TRAFO-MAGNETVOIMENDI TUURKARAKTERISTIKUD}

On näidatud, et ühefaasiline trafo-magnetvõimendi on teatud tingimustel kiiretoimeline, ning leitud tema tüürkarakteristikud küllastamata südamiku induktiivsust arvestades.

V. KAASIK, J. SOOJARV, J. HUNT, T. LAUSMAA

\section{CONTROL CHARACTERISTICS OF SINGLE-PHASE DIFFERENTIAL TRANSFORMER MAGNETIC AMPLIFIER}

It is shown that under certain conditions the single-phase differential-transformer magnetic amplifier has a basic half-cycle response; taking into account the inductance in the unsaturated core, its control characteristics are calculated. 